\title{
Bloqueo nervioso corticoideo-bupivacaína: ¿La mejoría post-procedimiento inmediato predice el éxito terapéutico a mediano plazo?
}

\section{Corticoid-bupivacaine Nerve Block: Does the Immediate Relief Postprocediment Predicts Medium-term Therapeutic Success?}

\author{
Ricardo Cobeñas ${ }^{1}$ Juan Flórez ${ }^{1}$ Pablo Chiaradia11 \\ Mariángela Paba ${ }^{1}$ Jairo Hernández Pinzón ${ }^{1,20}$

\footnotetext{
1 Departamento de Imágenes, Centro de Educación Médica e Investigaciones Clínicas (CEMIC), Hospital Universitario Sede Saavedra, Ciudad Autónoma de Buenos Aires, Argentina

2 Instituto de Investigaciones Neurológicas, Dr. Raúl Carrea (FLENI), Ciudad Autónoma de Buenos Aires, Argentina
}

Rev Argent Radiol 2021;85:91-97.
Kelly Ruiz Salgado ${ }^{1}$ María De Vedia ${ }^{1}$

Address for correspondence Jairo Hernández Pinzón, (e-mail: jairohernandez1@hotmail.com).

\begin{abstract}
Resumen
Palabras clave

- Bloqueo nervioso

- anestesia epidural

- dolor

- tomografía computada

Objetivo Establecer la relación entre la reducción inmediata de los síntomas post bloqueo nervioso lumbar guiado por tomografía computada (TC) y la mejoría tras un lapso de tiempo de un mes.

Materiales y métodos Se analizaron datos sobre 46 procedimientos de bloqueos lumbares radiculares y epidurales guiados por TC realizados entre diciembre de 2018 y marzo de 2019. Los pacientes firmaron consentimiento informado y el Comité de Ética en Investigación de nuestra institución aprobó el estudio. Se colectó, mediante un cuestionario dicotómico, información acerca de los síntomas iniciales, los cambios inmediatos al procedimiento y los síntomas tras un mes del mismo.

Resultados Inmediatamente post-bloqueo, 30 pacientes (65\%) mostraron mejoría o ausencia de síntomas y seis de ellos manifestaron persistencia de los síntomas al mes. Post-procedimiento inmediato, 16 pacientes (34\%) no presentaron ningún cambio en la sintomatología. De estos, siete presentaron mejoría al mes. La razón de momios para la persistencia del efecto analgésico al mes en los casos en los que el paciente manifestó alivio inmediato después del procedimiento fue de 5,1 (95\% IC 1.128 a 24.031). La prueba de McNemar para determinar si esta diferencia en la proporción de pacientes con alivio inmediato posterior a la punción y persistencia del efecto analgésico arrojó un p value de 0,08.
\end{abstract}

received

August 16, 2019

accepted

October 14, 2020

published online

February 4, 2021
DOI https://doi.org/

$10.1055 / \mathrm{s}-0040-1721349$.

ISSN 1852-9992. (C) 2021. Asociación Civil Sociedad Argentina de Radiología and
Federacion Argentina de Asociaciones de Radiología, Diagnóstico
por Imágenes y Terapia Radiante. All rights reserved.
This is an open access article published by Thieme under the terms of the
Creative Commons Attribution-NonDerivative-NonCommercial-License,
permitting copying and reproduction so long as the original work is given
appropriate credit. Contents may not be used for commercial purposes, or
adapted, remixed, transformed or built upon. (https://creativecommons.org/
licenses/by-nc-nd/4.0/)
Thieme Revinter Publicações Ltda., Rua do Matoso 170, Rio de
Janeiro, RJ, CEP 20270-135, Brazil 


\author{
Abstract \\ Keywords \\ - nerve block \\ - Epidural Anesthesia \\ - pain \\ - computed \\ tomography
}

Conclusión Existió asociación positiva entre la mejoría inmediata y la ausencia de síntomas al mes. Sin embargo, el análisis estadístico de antes y después insinúa que estos resultados pudieron ser por el azar.

Objective To establish the relationship between the immediate symptom reduction of post-lumbar nerve block guided by Computed Tomography (CT) and the improvement after a period of one month.

Materials and methods Data from 46 patients who performed lumbar, foraminal and epidural CT-guided blocks were analyzed, performed from December 2018 to March 2019. The patients signed a written informed consent and our institutional Ethical Committee approved the study. By means of a dichotomous questionnaire, information was collected about the initial symptoms, the immediate changes after the procedure and the presence of symptoms one month later.

Results Immediately after blockade, 30 patients (65\%) showed improvement or absence of symptoms six of them manifested persistence of symptoms after a month. Immediately after the procedure, $16(34 \%)$ patients did not present any change in the symptomatology. Seven of them showed improvement after a month. Odds ratio for persistence of analgesic effect after a month when the patient had manifested immediate relief after procedure was 5.1 (95\% IC 1.12824.031). Nevertheless, exact Mc Nemar's test to establish statistically significant difference in patients with immediate relief and those with persistence of it after a month showed a $\mathrm{p}$ value of 0.08 .

Conclusion There was a positive association between immediate and midterm symptom relief, however before and after statistical analysis hints that this results may have been given by chance.

\section{Introducción}

El dolor lumbar crónico es uno de los principales motivos de ingreso en los servicios de urgencias, así como también en la consulta médica general y especializada. Entre el 70 y el $80 \%$ de la población lo padece en algún momento de su vida y en Estados Unidos es la causa más frecuente de limitación funcional en mayores de 45 años. ${ }^{1}$ La patología vertebral y discal es una de las principales causas de dolor lumbar ya que puede generar compresión mecánica de las raíces nerviosas, esto desencadena una cascada inflamatoria en la cual citoquinas proinflamatorias como las interleucinas 1, 6, 8 y el factor de necrosis tumoral generan una disminución del flujo sanguíneo, originando coagulación intravascular y disminución de la velocidad de conducción nerviosa, lo que afecta la transmisión de los nervios espinales y genera un dolor con patrón dermatomérico (dolor radicular). ${ }^{1,2}$

En 1930 se describió por primera vez la analgesia epidural para el manejo del dolor lumbar y 40 años después es un método fundamental para el manejo de pacientes con dolor lumbar de origen radicular. Durante estas décadas, las punciones analgésicas se realizaban de acuerdo a ciertos puntos de referencia topográficos y fue alrededor de 1980 que los radiólogos empezaron a utilizar la fluoroscopía como guía para determinar la posición de las agujas. ${ }^{3-6}$
En las décadas de 1980 y 1990 se incrementó significativamente el número de procedimientos intervencionistas para el manejo del dolor lumbar. Estados Unidos fue un modelo de esto, reportando hasta 2010 más de 2,2 millones de punciones lumbares para el manejo del dolor. ${ }^{7,8}$ Se calcula que el gasto en salud por este método tuvo un incremento del $629 \%$ entre 1994 y $2001 .^{9}$

El uso excesivo de esta técnica ha conducido al escrutinio de sus resultados terapéuticos y gran parte de los artículos reportados sobre este tema arrojan contradicciones. Por ejemplo, los análisis epidemiológicos se centran en hallazgos a largo plazo y puntos finales quirúrgicos, mientras que los artículos escritos por radiólogos intervencionistas que realizan estos procedimientos se centran en resultados a corto y mediano plazo. $^{10}$

Nuestra hipótesis consiste en que la mejoría inmediata post-procedimiento puede predecir el éxito terapéutico a mediano plazo ya que, al finalizar el procedimiento, una mejora en los síntomas sugiere que, al estar haciendo efecto la bupivacaína y esta haber sido administrada en conjunto con el corticoide, el sitio de infiltración elegido es el correcto, el procedimiento fue técnicamente adecuado y debería hacer efecto a mediano-largo plazo. Por lo tanto, el objetivo de esta investigación es reportar resultados en cuanto a mejoría o persistencia de síntomas en una serie de pacientes que 
fueron sometidos a bloqueos analgésicos guiados por tomografía computada (TC) en nuestra institución.

\section{Materiales y métodos}

Previa aprobación por el Comité de Ética de nuestro Centro, se llevó a cabo un estudio observacional prospectivo en pacientes con dolor espinal crónico que fueron sometidos a bloqueos lumbares radiculares y epidurales guiados por TC entre diciembre de 2018 y marzo de 2019 en nuestra institución.

Todos los procedimientos fueron realizados por el mismo médico especialista en diagnóstico por imágenes, con 8 años de experiencia en intervencionismo percutáneo (RC).

Todos los pacientes firmaron consentimiento informado e inmediatamente luego de realizarse el procedimiento fueron interrogados por el médico intervencionista acerca de los cambios con respecto a la sintomatología inicial. Se realizó un cuestionario dicotómico, en el que se consideró como afirmativo a una disminución o ausencia del dolor inicial y como negativo a una persistencia o aumento en el dolor. Luego, un mes post-procedimiento, fueron entrevistados telefónicamente en cuanto a la persistencia o mejoría de los síntomas por médicos residentes de segundo y cuarto año en diagnóstico por imágenes, los cuales se encontraban rotando por la subespecialidad de intervencionismo percutáneo utilizando el mismo método dicotómico anteriormente descripto. Todos los datos fueron recolectados en una hoja de Microsoft Excel.

Los criterios de inclusión fueron: pacientes mayores de 18 años, que se realizaron bloqueos lumbares espinales 0 radiculares, con tolerancia al decúbito prono y test de coagulación dentro de límites normales.

Los criterios de exclusión fueron: pacientes con varios niveles afectados, con antecedentes de bloqueos previos, así como también aquellos que hubieran sido sometidos a cirugías u otros tratamientos en el periodo de observación.

Para realizar el procedimiento se ubicaron a los pacientes en un tomógrafo Philips MX (Cleveland, Ohio, USA) de 16 detectores en decúbito prono o lateral, según tolerancia. Los parámetros de adquisición de las imágenes fueron $1 \mathrm{~mm}$ cada 0,5 mm de espesor, FOV 340-450, matriz de 512, Kv $80 \mathrm{~mA}$ 120; se colocó un marcador metálico a nivel dorsal, en la piel y mediante TC se identificó la raíz nerviosa afectada o el lugar de la estenosis; luego se determinó el ángulo de enfoque y se marcó con tinta indeleble la zona cutánea de punción.

Se utilizó iodopovidona para realizar la asepsia y se aplicó anestesia local con lidocaína al $2 \%$ para disminuir las molestias causadas por el procedimiento. Se introdujo una aguja espinal $21 \mathrm{G}$ hasta el lugar afectado fuera radicular o epidural ( - Fig 1.). Una forma de asegurar que se alcanzó la zona indicada en el caso de los bloqueos radiculares fue la reproducción del dolor referido por el paciente durante el interrogatorio, lo que se interpretó como contactando en el nervio comprometido (-Fig 2). En bloqueos epidurales previo a la aplicación del medicamento, se inyectó $1 \mathrm{~cm}^{3}$ de aire para confirmar que la aguja se encontraba en el espacio correcto. En todos los casos se inyectó una solución compuesta de $30 \mathrm{mg}$ de triamcinolona y 1,5 $\mathrm{mg}$ de clorhidrato de bupivacaina.

Para el análisis estadístico se utilizó el sofware Stata (Versión 15.0, StataCorp, CollegeStation, Texas), se

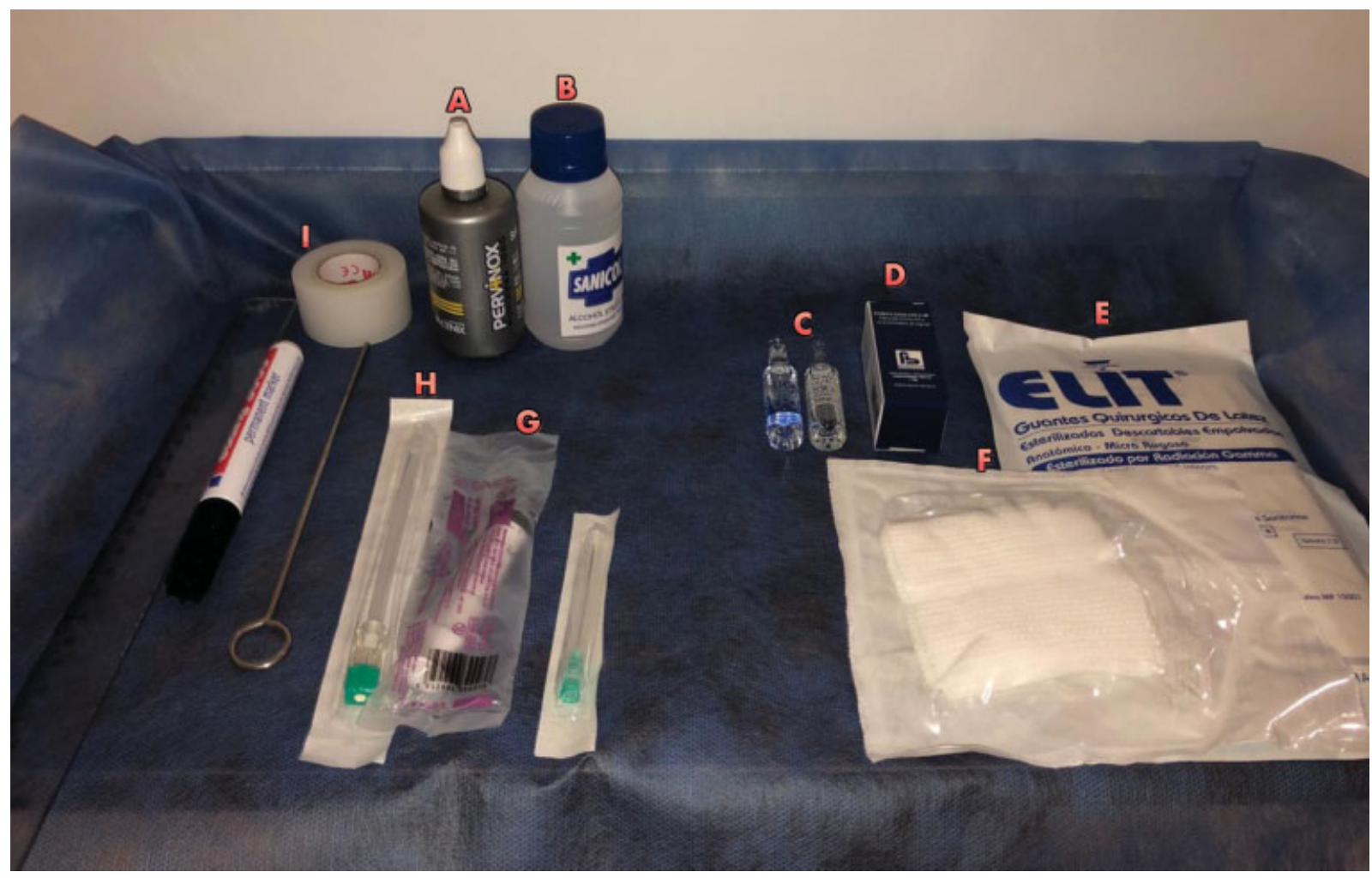

Fig. 1 Materiales utilizados en el procedimiento de bloqueo nervioso epidural y radicular. (A) lodopovidona. (B) Alcohol. (C) Lidocaína 2\%. (D) Triamcinolona $30 \mathrm{mg}$. (E) Guantes estériles. (F) Gasas. (G) Jeringa $10 \mathrm{ml}$. (H) Aguja espinal 21 G. (I) Cinta adhesiva. 


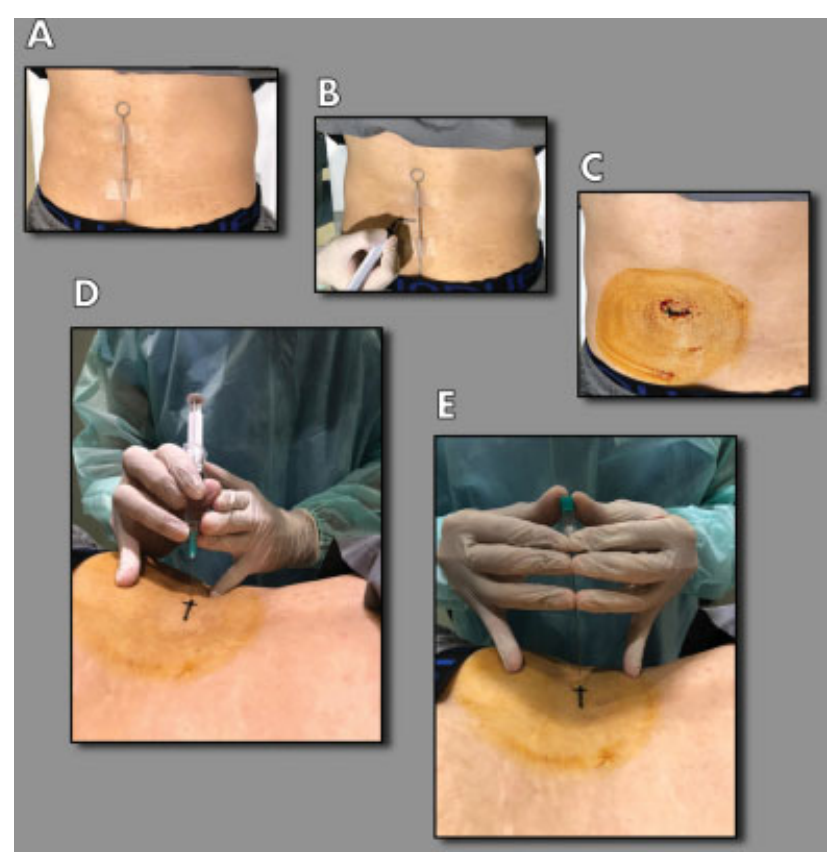

Fig. 2 Técnica de punción. (A) Se ubica al paciente en decúbito prono y se coloca un marcador metálico a nivel dorsal en la piel. (B) Mediante TC se identifica la raíz nerviosa afectada y se marca con tinta indeleble la zona cutánea de punción. (C) Se realiza antisepsia con iodopovidona. (D) Se aplica anestesia local con lidocaína al 2\%. (E) Se introduce una aguja espinal $21 \mathrm{G}$ hasta el lugar afectado donde, luego de verificar mediante TC, se inyecta una solución compuesta de $30 \mathrm{mg}$ de triamcinolona y $1,5 \mathrm{mg}$ de clorhidrato de bupivacaina.

reportaron los datos demográficos básicos mediante estadígrafos de tendencia central y dispersión, se usó la razón de momios como medida de asociación y la prueba de McNemar para testear nuestra hipótesis.

Para evaluar si existió asociación entre la mejoría de los síntomas en el postrpocedimiento inmediato y tardío con el sexo del paciente se realizó una prueba exacta de Fisher. También, mediante una prueba de T de Student, se buscó evaluar la relación entre la mejoría de los síntomas en ambas etapas mencionadas y la edad del paciente.
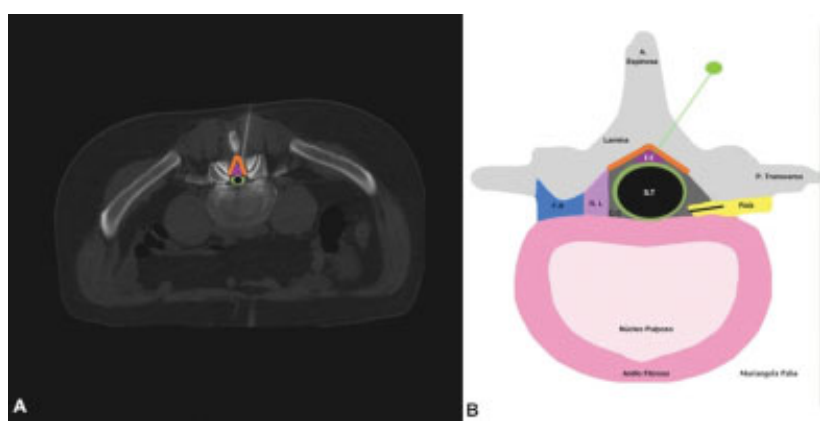

Fig. 3 (A) TC sin contraste, corte axial. Bloqueo epidural a nivel de L5S1 izquierdo. Paciente en decúbito prono. Luego de la aplicación de aire y de asegurarnos de la ubicación adecuada en el espacio epidural, se procedió al pasaje de la medicación. (B) Esquema en el que se detalla la anatomía regional. EE: espacio epidural; ST: saco tecal; borde verde: dura madre; CC: canal central; RL: receso lateral; FN: foramen neural; líneas naranjas: ligamento amarillo.
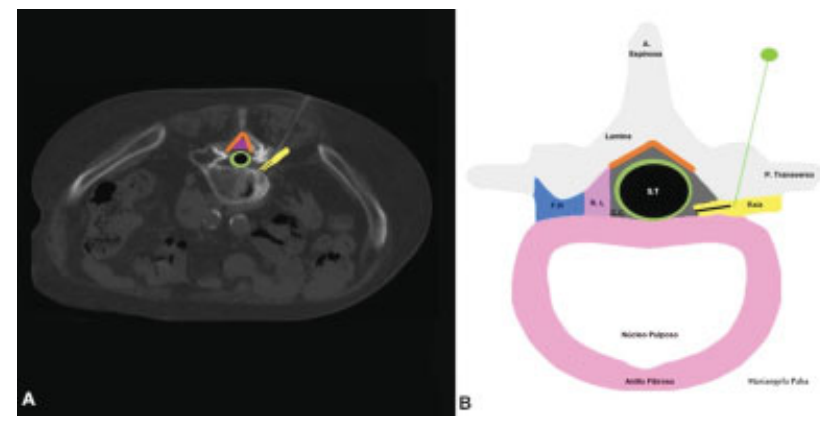

Fig. 4 (A) TC sin contraste, corte axial. Bloqueo selectivo foraminal izquierdo a nivel de $L 5$ izquierda, paciente en decúbito prono. Se observa la aguja con su extremo en contacto con la raíz. (B) Esquema donde se detalla la anatomía regional. S.T: saco tecal; borde verde: dura madre; CC: canal central; RL: receso lateral; FN: foramen neural; líneas naranjas: ligamento amarillo.

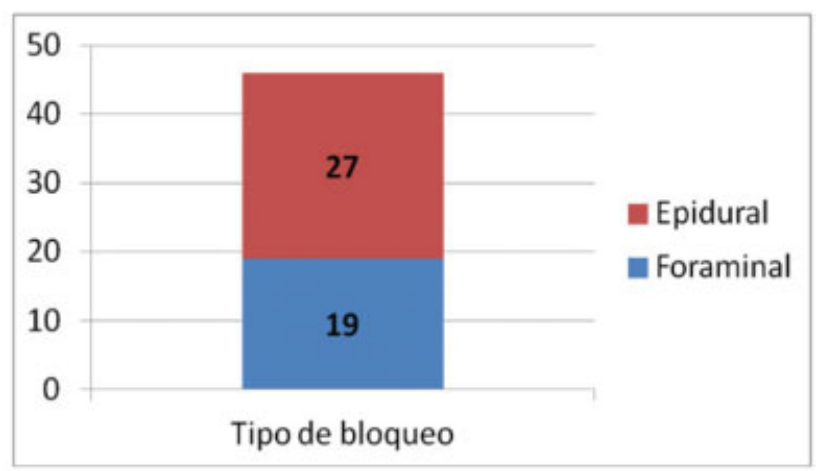

Gráfico 1 Porcentaje de los procedimientos realizados a los pacientes con dolor radicular crónico.

\section{Resultados}

En total se estudiaron 46 pacientes, 34 mujeres $(73,9 \%)$ y 12 hombres (26\%) con una edad media de 67 años $+/$ - 11,7 años (rango 33 a 89 años). En 27 (58\%) de ellos se realizó bloqueo epidural (-Fig. 3) y en 19 (41\%) bloqueo radicular (-Fig. 4) (-Gráfico 1).

Del total de pacientes, 30 (65\%) reportaron mejoría inmediata post-procedimiento y $16(34 \%)$ no lo hicieron (-Gráfico 2). De estos últimos, solo 9 presentaron dolor al mes.

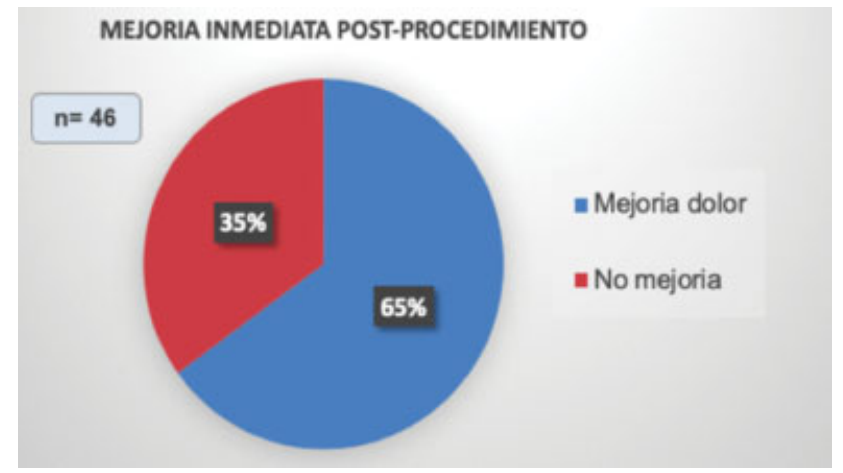

Gráfico 2 Gráfico de torta que muestra la proporción de pacientes que obtuvieron una mejoría o disminución post-procedimiento. 
Tabla 1 Tabla de contingencia que muestra cantidad de pacientes que presentaron mejoría de los síntomas post-procedimiento inmediato y mejoría de los síntomas a mediano plazo

\begin{tabular}{|l|l|l|}
\hline Tipo de Bloqueo & Epidural & Radicular \\
\hline $\begin{array}{l}\text { Mejoría inmediata } \\
\text { Post-procedimiento. }\end{array}$ & 20 & 10 \\
\hline Mejoría a mediano plazo & 19 & 12 \\
\hline
\end{tabular}

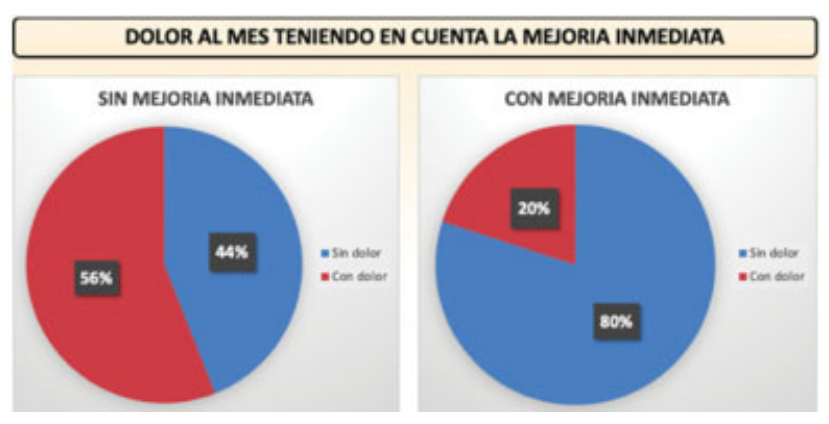

Gráfico 3 Gráfico de torta que representa la proporción de pacientes sintomáticos al mes, teniendo en cuenta las variables: sin mejoría inmediata/con mejoría inmediata post-procedimiento.

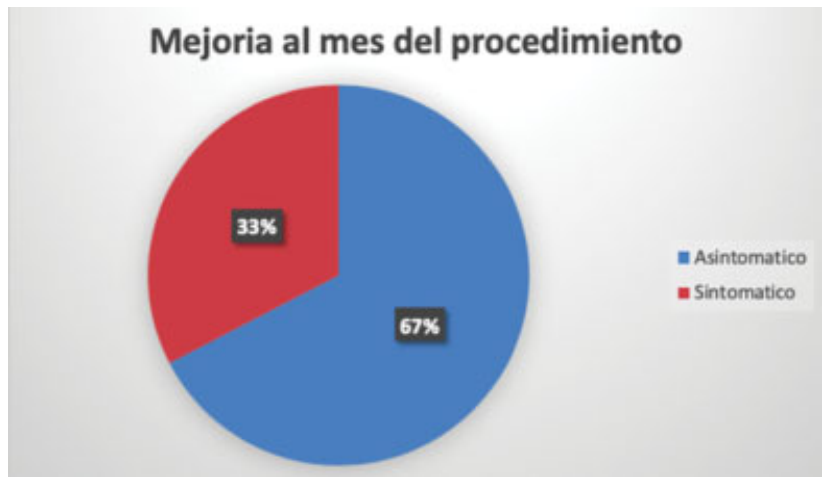

Gráfico 4 Gráfico de torta que representa el porcentaje de pacientes con mejoría al mes del procedimiento y los que presentaron dolor en ese lapso de tiempo.

Dentro del grupo de 27 pacientes con bloqueos epidurales, 20 mostraron mejoría inmediata y 19 tardía, observando proporciones de mejoría similares al mes de realizado el procedimiento (74\% y 70\% respectivamente). En el grupo de los pacientes con bloqueos raquídeos, 10 mostraron mejoría inmediata y 12 tardía, observando también similares

Tablas 2 Diferencia de frecuencias y porcentajes de pacientes con mejoría inmediata y tardía de acuerdo al sexo

\begin{tabular}{|l|l|l|l|}
\hline Mejoría Inmediata & Femenino & Masculino & Total \\
\hline No & $13(81,2 \%)$ & $3(18,7 \%)$ & 16 \\
\hline Si & $21(70 \%)$ & $9(30 \%)$ & 30 \\
\hline Total & 34 & 12 & 46 \\
\hline Fisher's exact $=0,498$ \\
\hline
\end{tabular}

Tablas 3 Diferencia de frecuencias y porcentajes de pacientes con mejoría inmediata y tardía de acuerdo al sexo

\begin{tabular}{|l|l|l|l|}
\hline Mejoría Tardía & Femenino & Masculino & Total \\
\hline No & $13(86,67 \%)$ & $2(13,33 \%)$ & 15 \\
\hline $\mathrm{Si}$ & $21(67,74 \%)$ & $10(32,26 \%)$ & 31 \\
\hline Total & 34 & 12 & 46 \\
\hline \multicolumn{4}{|l}{ Fisher's exact $=0,285$} \\
\hline
\end{tabular}

Tablas 4 Edad media de los pacientes con y sin mejoría de los síntomas post procedimiento inmediato vs edad media de los pacientes con y sin mejoría tardía de los mismos

\begin{tabular}{|l|l|l|}
\hline $\begin{array}{l}\text { Edad media en pacientes } \\
\text { con mejoria inmediata }\end{array}$ & & $\begin{array}{l}\text { Edad media en pacientes } \\
\text { sin mejoria inmediata }\end{array}$ \\
\hline $65,7+/-12,9$ & $\mathrm{v} / \mathrm{s}$ & $71,8+/-7,7$ \\
\hline $\mathrm{p}=\mathbf{0 , 9 5 4}$ & \\
\hline
\end{tabular}

Tablas 5 Edad media de los pacientes con y sin mejoría de los síntomas post procedimiento inmediato vs edad media de los pacientes con y sin mejoría tardía de los mismos

\begin{tabular}{|l|l|l|}
\hline $\begin{array}{l}\text { Edad media en pacientes } \\
\text { con mejoria tardia }\end{array}$ & & $\begin{array}{l}\text { Edad media en pacientes } \\
\text { con mejoria tardia }\end{array}$ \\
\hline $67,54+/-12,8$ & $\mathrm{v} / \mathrm{s}$ & $68,4+/-9,4$ \\
\hline $\mathrm{P}=\mathbf{0 , 5 9 0}$ & \\
\hline
\end{tabular}

proporciones de mejoría al mes de realizado el procedimiento (52\% y 63\% respectivamente) (- Tabla 1 ).

De los 30 pacientes que presentaron mejoría inmediata, 6 de ellos (20\%) manifestaron persistencia de los síntomas iniciales al mes y los restantes 24 (80\%) se mantuvieron sin sintomatología en dicho período. De los 16 pacientes que no tuvieron mejoría inmediatamente, 7 (44\%) reportaron mejoras al mes (-Gráficos 3 y 4).

En las -Tablas 2 y $\mathbf{3}$ se muestra la diferencia de frecuencias $\mathrm{y}$ porcentajes de pacientes con mejoría inmediata y tardía de acuerdo al sexo. No se evidenciaron diferencias estadísticamente significativas entre ambos grupos (Fisher exact test $=0,0498$ y Fisher exact test $=$ 0,285 respectivamente).

En cuanto a la edad de los pacientes con mejoría inmediata y tardía versus los pacientes sin mejoría inmediata ni tardía, tampoco se encontraron diferencias estadísticamente significativas $(64,7+/-12,9$ años v/s 71,8 +/- 7,7 años, $t$ (44): $-1,7289$, p-value $=0,954)$ y $(67,5+/-12,8$ años v/s 68,4 $+/-9,4$ años, $t(44):-0,23, \mathrm{p}$-value $=0,59)$ respectivamente (-Tablas 4 y 5 ).

La razón de momios para la persistencia del efecto analgésico al mes en los casos en los que el paciente manifestó alivio inmediato después del procedimiento fue de 5,1 (95\% IC 1.128 a 24.031). Sin embargo, la prueba de McNemar para determinar si esta diferencia en la proporción 
de pacientes con alivio inmediato posterior a la punción y persistencia del efecto analgésico al mes pudo ser a causa del azar, arrojó un p-value de 0,08.

\section{Discusión}

Los bloqueos radiculares y facetarios son procedimientos realizados desde hace ya varias décadas para el manejo del dolor en las radiculopatías y enfermedades facetarias, esto ha conllevado a la reducción del consumo de analgésicos, mantenimiento de las actividades laborales y eliminación de la necesidad de una cirugía en muchos individuos. ${ }^{11}$

La sensibilidad de los bloqueos ha aumentado notoriamente al incorporarse una guía fluoroscópica o tomográfica ya que se puede identificar de forma precisa reparos anatómicos y medir la profundidad necesaria para alinear la aguja hacia la zona radicular o epidural. ${ }^{11}$

La literatura aún no provee evidencia acerca de la eficacia de los bloqueos nerviosos. Los resultados en el uso de esteroides y anestésicos a nivel epidurales y radiculares son cuestionables dada la falta de control y de calidad en la mayoría de los estudios. En los diferentes estudios realizados, se reportan porcentajes de éxito que van del 45 al 100\%. Esta variabilidad depende de la metodología y técnica utilizada, el resultado evaluado y la selección de pacientes. ${ }^{12}$

Las causas de la diferencia en los resultados en los estudios se deben a la falta de guías que avalen dosis, tipo de esteroide, frecuencia de administración y volumen a inyectar, además en gran parte de los artículos públicos la mayoría de estos procedimientos analgésicos se realizaron sin guía imagenológica. ${ }^{1}$

En el conocimiento de los autores, no hay en la bibliografía trabajos que relacionen la mejoría inmediata de los síntomas con el éxito terapéutico a largo plazo y vale la pena destacar que en nuestro medio existen pocos reportes sobre este tema con seguimiento de pacientes a mediano plazo, por lo cual es de nuestro interés compartir nuestros resultados.

Nuestro estudio demostró una reducción de la sintomatología en un $63 \%$ de los pacientes en el postprocedimiento inmediato y en un $67 \%$ al mes de realizado el mismo (-Gráfico 4). Esto va en consonancia con lo reportado por autores como Boswell y col, ${ }^{13}$ que en 2003 publicaron una revisión sistemática donde encontraron que en tres estudios randomizados se concluyó que existía una fuerte evidencia de efectividad de los bloqueos foraminales en pacientes con dolor lumbar y evidencia moderada para bloqueos epidurales. Se llegó a similares conclusiones a partir de un análisis de estudios randomizados y de cohorte prospectivo en el año 2007 por Young y col. ${ }^{14}$ También Watts R. y Silagy C, ${ }^{15}$ en un meta-análisis de 11 trabajos aleatorizados con 907 pacientes, demostraron una mejoría de más del 75\% en la reducción del dolor tanto a corto (1-60 días) [razón de momios (OR)2.61 Intervalo de confianza (IC) $95 \% 1,90$ a 3,77], como a largo plazo (12 semanas - 1 año) OR 1.87-IC 95\% 1,31 a 2,68), concluyendo que esa técnica es eficaz en el tratamiento del dolor lumbar de origen radicular.

No obstante, hay que dejar claro que, al testear nuestra hipótesis, encontramos la posibilidad de que la proporción de pacientes con mejoría inmediata y sostenida a mediano plazo haya sido a causa del azar.

Otros autores, como De Palma y col. ${ }^{16}$ reportan, mediante una revisión crítica de seis artículos, que la evidencia de efectividad de este procedimiento es moderada y Adigüzel y col. ${ }^{17}$ informó, en su serie de 62 pacientes, que la provocación del dolor en el momento de la infiltración anestésica no está relacionada con la mejoría a largo plazo. Sin embargo, la punción radicular fue efectiva en el corto y mediano plazo (puntaje medio de la escala visual análoga de 80 postprocedimiento v/s 45 a las dos semanas v/s 30 a las 12 semanas p-value: $<0.001)$.

Es importante mencionar que las punciones lumbares para el manejo del dolor son procedimientos con una incidencia de complicaciones entre el 0,07 y $0,5 \%$ (las cuales se pueden dar por el procedimiento en sí o por los medicamentos utilizados), dentro de las que se destacan cefalea post-punción entre el 7,5\% y el 75\% dependiendo de variables como técnica, tamaño de la aguja y experiencia del médico que realiza el procedimiento; infección en el 1\%, donde la principal comorbilidad asociada para desarrollarla es diabetes mellitus; debilidad motora transitoria, síndrome de cauda equina (muy pocos casos reportados), con resolución de los síntomas en un período de horas o días y daño neurológico directo; entre otras. ${ }^{18,19}$ En nuestro estudio, no se reportaron complicaciones destacables inmediatas ni posteriores al procedimiento y creemos que puede estar relacionado al uso de guía tomográfica y a la experiencia del médico intervencionista.

Encontramos como limitaciones de este trabajo el bajo tamaño muestral y la posible heterogeneidad en cuanto a las características basales de los pacientes. Tampoco se tuvieron en cuenta algunas covariables que pueden estar íntimamente relacionadas con los resultados, como causa del dolor, localización y magnitud de las discopatías y el peso del paciente, entre otras. Tampoco se realizó un seguimiento a largo plazo, lo que limita en gran medida la posibilidad de establecer conclusiones categóricas de nuestros resultados.

\section{Conclusión}

En nuestra serie de casos encontramos un aumento en las chances de mostrar mejoría en mediano plazo en los pacientes cuando estos presentaron mejoría inmediata post-punción. Sin embargo, el análisis estadístico de antes y después insinúa que estos resultados pudieron ser a causa del azar.

Responsabilidades Éticas

Protección de personas y animales. Los autores declaran que para esta investigación no se han realizado experimentos en seres humanos ni en animales.

Confidencialidad de los datos. Los autores declaran que han seguido los protocolos de su centro de trabajo sobre la publicación de datos de pacientes.

Derecho a la privacidad y consentimiento informado. Los autores declaran que en este artículo no aparecen datos de pacientes. 


\section{Conflictos de intereses}

Los autores declaran tener los siguientes conflictos de intereses:

Ricardo Cobeñas es revisor de la RAR.

Jairo Hernández Pinzón es miembro del Comité Editorial de la RAR.

\section{Bibliografía}

1 Álvarez Correa A, Rivera Díaz RC, Arcila Lotero MA. Eficacia de la metilprednisolona epidural en el dolor radicular. Rev Colomb Anestesiol. 2015;43(01):61-67

2 Cosamalón-Gan I, Cosamalón-Gan T, Mattos-Piaggio G, Villar-Suárez V, García-Cosamalón J, Vega-Álvarez JA. Inflamación en la hernia del disco intervertebral. Neurocirugía web site. http://www. revistaneurocirugia.com/es-inflamacion-hernia-del-discointervertebral-avance-S1130147320300026. Accessed April 21, 2020

3 Evans W. Intrasacral epidural injection in the treatment of sciatica. Lancet. 1930;2:1225-1229

4 Coomes EN. A comparison between epidural anaesthesia and bed rest in sciatica. BMJ. 1961;1(5218):20-24

5 Goebert HW Jr, Jallo SJ, Gardner WJ, Wasmuth CE, Bitte EM. Sciatica: treatment with epidural injections of procaine and hydrocortisone. Cleve Clin Q. 1960;27:191-197

6 el-Khoury GY, Ehara S, Weinstein JN, Montgomery WJ, Kathol MH. Epidural steroid injection: a procedure ideally performed with fluoroscopic control. Radiology. 1988;168 (02):554-557

7 Manchikanti L, Pampati V, Boswell MV, Smith HS, Hirsch JA. Analysis of the growth of epidural injections and costs in the Medicare population: a comparative evaluation of 1997, 2002, and 2006 data. Pain Physician. 2010;13(03):199-212

8 Manchikanti L, Pampati V, Falco FJE, Hirsch JA. Assessment of the growth of epidural injections in the medicare population from 2000 to 2011. Pain Physician. 2013;16(04): E349-E364
9 Friedly J, Chan L, Deyo R. Increases in lumbosacral injections in the Medicare population: 1994 to 2001. Spine. 2007;32(16):1754-1760

10 Palmer WE. Spinal Injections for Pain Management. Radiology. 2016;281(03):669-688

11 González FJ, Adén M. Bloqueo radicular lumbar selectivo guiado por TAC. Alternativa para diagnóstico y tratamiento de radiculopatías originadas por hernias discales y/o artrosis facetarías. Rev Fac Med (Caracas). 2011;31(02):11-14

12 Nielsen AJ, Criscuolo G, González Calvo S, Larrañaga N, Gallo JC, Kozima S. Bloqueo nervioso lumbar selectivo guiado por tomografía computada. Nuestra experiencia en un hospital universitario. Rev Argent Radiol. 2013;77(03):226-230

13 Boswell MV, Hansen HC, Trescot AM, Hirsch JA. Epidural steroids in the management of chronic spinal pain and radiculopathy. Pain Physician. 2003;6(03):319-334

14 Young IA, Hyman GS, Packia-Raj LN, Cole AJ. The use of lumbar epidural/transforaminal steroids for managing spinal disease. J Am Acad Orthop Surg. 2007;15(04):228-238

15 Watts RW, Silagy CA. A meta-analysis on the efficacy of epidural corticosteroids in the treatment of sciatica. Anaesth Intensive Care. 1995;23(05):564-569

16 DePalma MJ, Bhargava A, Slipman CW. A critical appraisal of the evidence for selective nerve root injection in the treatment of lumbosacral radiculopathy. Arch Phys Med Rehabil. 2005;86(07): 1477-1483

17 Adıgüzel E, Tecer D, Güzelküçük Ü, Taşkaynatan MA, Tan AK. The effectiveness of transforaminal epidural steroid injection in patients with radicular low back pain: Combination of pain provocation with effectiveness results. Turk J Phys Med Rehabil. 2017;63(02):117-123

18 Chou R. Subacute and chronic low back pain: Nonsurgical interventional treatment. SOMEPOMED Website http:// somepomed.org/articulos/contents/mobipreview.htm?36/15/ 37104. Updated September 30, 2011. Accessed April 21, 2020

19 Murad P, Baltazar A, Zabala ME, Iñiguez C, Martínez E, Mattanó J. Errores en el procedimiento del bloqueo epidural. Rev Argent Radiol. 2015;79(03):139-144 